\title{
Corona enhancement in ultrasonographical post-vascular phase images with microbubble contrast agent: A novel specific sign for hepatocellular carcinomas
}

\author{
KOUJI YAMAMOTO ${ }^{1}$, SHIGEO NAKANISHI ${ }^{1}$, HIROYUKI FUKE ${ }^{2}$, AKIRA HASHIMOTO $^{2}$, \\ ATSUYA SHIMIZU $^{2}$, TOSHINOBU HAMATAKI ${ }^{3}$ and KATSUYA SHIRAKI ${ }^{1}$ \\ ${ }^{1}$ First Department of Internal Medicine, Mie University School of Medicine, Tsu, Mie 514-8507; \\ ${ }^{2}$ Department of Internal Medicine, Saiseikai Matsusaka General Hospital of Medicine, Matsusaka, Mie 515-8557; \\ ${ }^{3}$ Sales Engineering Department, Toshiba Medical Systems Corp., Nagoya, Aichi 450-0003, Japan
}

Received September 26, 2005; Accepted November 21, 2005

\begin{abstract}
In 1.5 Harmonic Imaging ultrasonography (1.5 HI US), images are obtained in a band intermediate between the fundamental and 2nd harmonic components, resulting in stronger contrast enhancement than in conventional harmonic imaging. We attempted to assess the hemodynamics of hepatocellular carcinomas (HCC) with special attention to blood drainage using 1.5 HI US. Forty-two HCC nodules, metastatic liver tumors and hepatic hemangiomas were studied. In contrast studies, intermittent ultrasound transmission was performed for a period of up to $45 \mathrm{sec}$ after the injection of contrast agent, which was regarded as the vascular phase. The time point of 5 min later was specified as the post-vascular phase, and images were obtained by single manual transmission for comparison of contrast enhancement with surrounding hepatic parenchyma. In addition, histological examination was performed. 1.5 HI US clearly demonstrated the strong tumor vessels in most HCCs. Corona enhancement, in which the areas surrounding the tumor are enhanced, was observed in $71.4 \%$ (30/42) of HCC nodules during the post-vascular phase. This sign was not observed in any other tumors. Histological findings revealed that CD34-positive-endothelial cells were prominent in the surrounding area of HCC. In conclusion, 1.5 HI US is an effective tool for evaluating hemodynamics in both early- and post-vascular phase. Corona enhancement may be due to the trapping of contrast agent in the endothelial
\end{abstract}

Correspondence to: Dr Katsuya Shiraki, First Department of Internal Medicine, Mie University School of Medicine, 2-174 Edobashi, Tsu, Mie 514-8507, Japan

E-mail: katsuyas@clin.medic.mie-u.ac.jp

Abbreviations: HCC, hepatocellular carcinoma; $1.5 \mathrm{HI}, 1.5$ Harmonic Imaging

Key words: HCC, 1.5 Harmonic Imaging ultrasonography, corona enhancement, microbubble cells in the surround of HCC nodules and be a novel specific sign for HCC.

\section{Introduction}

The usefulness of the peripheral ultrasound contrast agent, Levovist, was initially thought to be limited simply to the enhancement of signals in color/power Doppler echo imaging $(1,2)$, but Levovist is currently also applied to the diagnosis of hepatic tumors. Levovist enhances blood flow signals and improves the visualization of tumor vessels in hepatic tumors (3-5). Recent technological advances in ultrasonographic diagnosis have led to the development of new imaging techniques that are collectively referred to as 'harmonic imaging', in which images are obtained using the 2nd harmonic components of the ultrasound signal. Among these new techniques, flash echo imaging (FEI) employs intermittent transmission at high acoustic power to allow the microbubbles to enter and fill the scanning plane, thus making it possible to obtain echoes with high sensitivity. In other words, FEI permits the acquisition of clear images with high spatial resolution in which only blood flow information is extracted, making it easier to visualize tumor vessels. In the contrast ultrasonographic examination of hepatocellular carcinoma (HCC), the microbubbles in Levovist cause higher frequency signals to be generated by the tumor than by normal hepatic parenchyma. Consequently, the contrast resolution of the tumor and normal parenchyma is further improved and minute blood vessels within the tumor can be visualized (6-16). In 2nd harmonic imaging $(2 \mathrm{HI})$, however, the presence of tissue harmonic components may lead to a reduction in contrast enhancement in some cases. To overcome this problem, echoes from tissue must be eliminated in order to extract only the echoes from the microbubbles. Subharmonic imaging and higher order harmonic imaging have been proposed, but subharmonic imaging suffers from low lateral resolution due to the low frequencies employed, while higher order harmonic imaging suffers from limited penetration due to the use of high frequencies, resulting in poor visualization of deep regions. In another method, Doppler technology is used to eliminate tissue echoes and visualize the irregular behavior of the microbubbles 
(17-19), but this method is susceptible to motion artifacts near the heart.

A new technology, 1.5 Harmonic Imaging ultrasonography (1.5 HI US), has been developed to overcome the above problems (20-23). In this method, images are obtained using a band that is intermediate between the fundamental and 2 nd harmonic components. In 1.5 HI US, a transmission waveform in which the bandwidth is limited is used, and the leakage of fundamental components is therefore reduced. The fundamental components of tissue echoes are effectively separated from the 2nd harmonic components, permitting images to be obtained in an intermediate band that is almost completely free from tissue echoes. When images are obtained in this intermediate band, higher contrast can be achieved between contrast agent and tissue echoes than in conventional $2 \mathrm{HI}$.

In the present study, we attempted to assess the hemodynamics of HCCs using 1.5 HI US. We found a novel diagnostic sign, corona enhancement in post-vascular phase images.

\section{Patients and methods}

Patients. The images evaluated in the present study were obtained from 42 nodules in 38 patients with HCC. The study group included 24 men and 14 women ranging in age from 41 to 78 years (mean age, 62 years). The minimum tumor diameter was $13 \mathrm{~mm}$ and the maximum tumor diameter was $42 \mathrm{~mm}$ (mean tumor diameter, $25.8 \pm 15.1 \mathrm{~mm}$ ). All 38 patients had hepatic cirrhosis and tested positive for the hepatitis $\mathrm{C}$ virus. There were 20 nodules for 20 cases of metastatic hepatic tumor consisting of 11 males and 19 females aged from 62 to 79 with an average age of 64 years, minimum tumor diameter of $25 \mathrm{~mm}$, maximum tumor diameter of $45 \mathrm{~mm}$, and average tumor diameter of $31.4 \pm 8.2 \mathrm{~mm}$. Seven cases had metastasis from stomach cancer, 11 cases had metastasis from bowel cancer and 2 cases had metastasis from lung cancer. There were also 8 nodules for 8 cases of hepatic hemangioma consisting of 5 males and 3 females aged from 32 to 56 with an average age of 45 years, minimum tumor diameter of $38 \mathrm{~mm}$, maximum tumor diameter of $56 \mathrm{~mm}$, and average tumor diameter of 44.6 $\pm 9.8 \mathrm{~mm}$. All cases were histopathologically diagnosed and examined using various diagnostic imaging techniques.

\section{Methods}

Contrast-enhanced ultrasonography. Ultrasound images were acquired using a diagnostic ultrasound system (SSA-770A, Aplio, Toshiba Medical Systems Corporation, Tokyo, Japan) with phased-array transducers operating at a frequency of $\sim 3.0 \mathrm{MHz}$ (PST-30AT sector transducer, Toshiba). The mechanical index (MI) was set to 1.6 , and frequencies of 2.3 MHz/3.3 MHz were employed in clinical $1.5 \mathrm{HI}$ US studies. Contrast images were acquired using FEI. In the clinical study, a total of $7 \mathrm{ml}$ of Levovist (Schering AG, Berlin, Germany) at a concentration of $300 \mathrm{mg} / \mathrm{ml}$ was injected as a single bolus via a peripheral vein at a rate of $1 \mathrm{ml} / \mathrm{sec}$. Images were acquired using FEI at intervals of $0.2 \mathrm{sec}$. The vascular phase was specified as the period up to $60 \mathrm{sec}$ after the injection of contrast agent, and the tumor vessels were observed with the focal point set at the bottom of the tumor. Then, after an interval of $4 \mathrm{~min}$, the post-vascular phase was specified as the period beginning at the 5-min time point, and ultra- sound transmission was performed once manually to observe the vascular enhancement (staining) of the tumor tissue. Images were recorded on S-VHS videotape immediately after the injection of contrast agent. The patient was instructed to hold his or her breath for 10-50 sec after the injection of Levovist in order to maintain a constant respiratory phase, and early-phase images were obtained continuously. Subsequently, the system was temporarily set to freeze mode and the images stored in cine loop memory were recorded on the system hard disk. At 5 min after injection, late-phase images were observed for several seconds during breath-holding and recorded on the system hard disk. The diagnostic results obtained using the various imaging modalities in the present study showed no variation between observers and no inconsistent cases.

Immunohistochemical staining for CD34 and CD68. Sections were deparaffinized and hydrated by passage through xylenes and a graded ethanol series (100\%-70\%). Antigen retrieval was performed on sections by incubation in $100 \mathrm{mM}$ sodium citrate ( $\mathrm{pH}$ 6.0). Sections were then treated with $0.3 \% \mathrm{H}_{2} \mathrm{O}_{2}$ in methanol for $30 \mathrm{~min}$ to eliminate endogenous peroxidase activity, and stained using the immunoperoxidase technique. Anti-CD34 antibody and anti-CD68 antibody in phosphatebuffered saline solution (PBS) containing normal goat serum were applied as primary antibodies for overnight, respectively. Secondary antibody application and peroxidase staining was performed using a biotin-conjugated goat anti-mouse secondary antibody and the $\mathrm{ABC}$ horseradish peroxidase method (Elite $\mathrm{ABC}$ kit, Vector Laboratories, $\mathrm{CA}$ ). After that, sections were washed, incubated with $0.05 \%$ diaminobenzidine $/ 0.15 \%$ $\mathrm{H}_{2} \mathrm{O}_{2}$, and counterstained with $10 \%$ hematoxylin (Waco Pure Chemical Industries, Osaka, Japan), respectively.

\section{Results}

1.5 HI US with Levovist in HCCs. We attempted to detect the blood flow within the tumor in 42 HCC nodules. Vascular phase $1.5 \mathrm{HI}$ US clearly demonstrated the tumor vessels from $20 \mathrm{sec}$ after contrast medium injection in 36 nodules $(85.7 \%)$. Furthermore, post-vascular phase images at 5 min after contrast medium injection revealed partial residual enhancement in most of the tumors, but with less enhancement than the surrounding hepatic parenchyma in all of the 42 nodules $(100 \%)$. Vascular phase images did not show hypervascular enhancement in 3 nodules $(14.3 \%)$, but post-vascular phase images demonstrated these 3 nodules as non-enhanced areas.

Surprisingly, contrast enhancement of adjacent liver was observed in 5 min after Levovist injection (Figs. 1 and 2). These, namely corona-like enhancement, in which the areas surrounding the tumor are strongly enhanced, was observed in $71.4 \%$ (30/42) (Table I). However, these corona enhancement was not detected in metastatic liver tumors and hepatic hemangiomas (Table II).

Histological examinations. In order to elucidate the mechanism of corona enhancement, we performed histological examinations in $7 \mathrm{HCC}$ nodules that were surgically resected. We found the presence of dilated portal vessels near the margins of the tumor (septal region). Furthermore, histological 


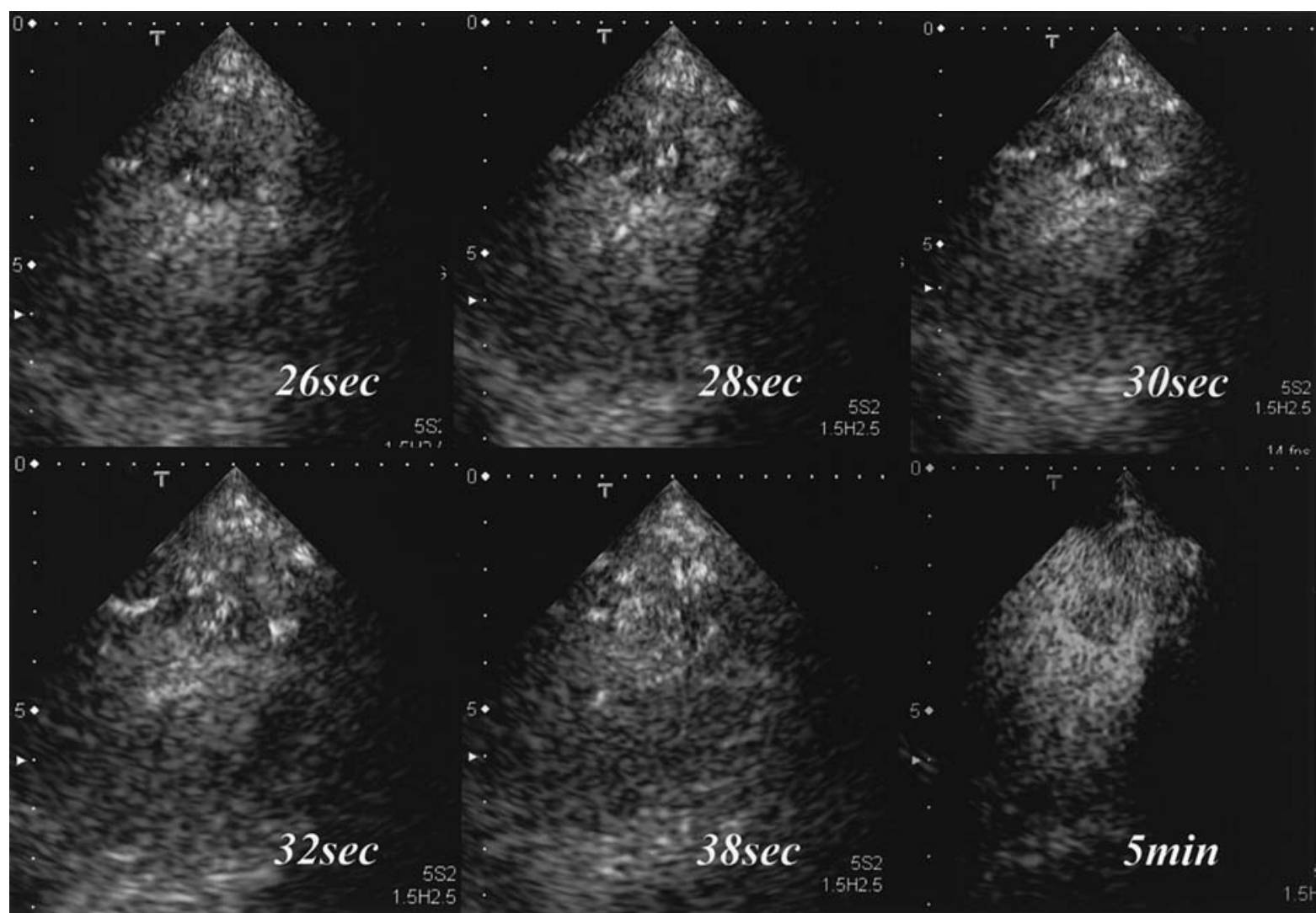

Figure 1. 1.5 HI images obtained from a 73-year-old man with HCC (diameter, $16.5 \mathrm{~mm}$ ). From the top row to the bottom row: pre-contrast and 26, 28, 30, $32,38 \mathrm{sec}$ and $5 \mathrm{~min}$ after contrast injection. The vessels in the tumor were depicted at $28 \mathrm{sec}$ after contrast injection and the entire tumor was enhanced with clearly visible margins at $38 \mathrm{sec}$. The contrast agent in the tumor was washed out at 5 min and the corona sign was clearly observed.

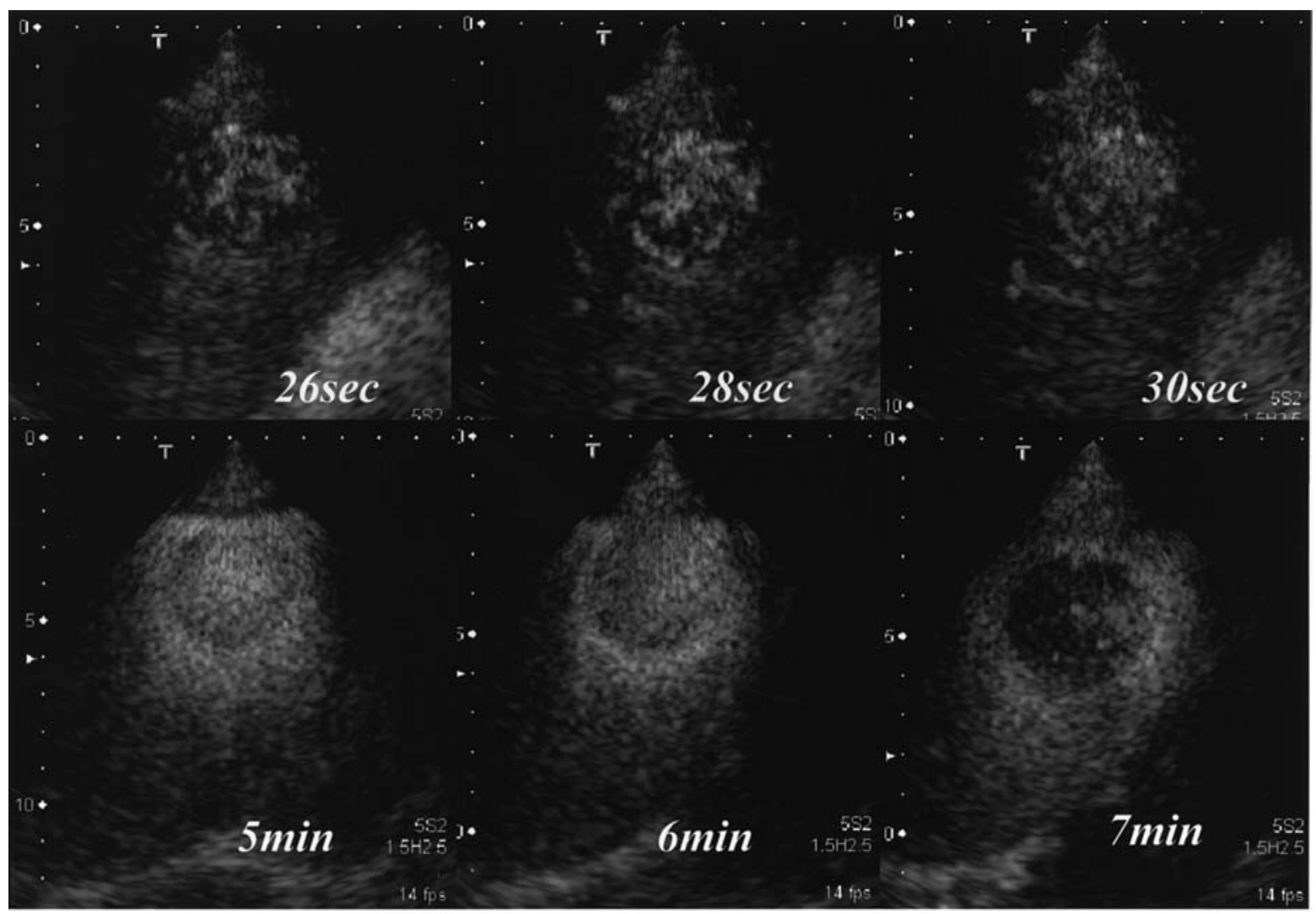

Figure 2. 1.5 HI images obtained from a 57-year-old man with HCC (diameter, $37.5 \mathrm{~mm}$ ). From the top row to the bottom row: pre-contrast and $26,28,30$ sec, 5,6 , and $7 \mathrm{~min}$ after contrast injection. The vessels in the tumor were depicted at $26 \mathrm{sec}$ and the entire tumor was enhanced with clearly visible margins at 30 sec. The contrast agent in the tumor was washed out at 5 and 6 min and the corona sign was clearly observed. Then, at 7 min, the corona sign became indistinct. 
Table I. Detection of HCC tumors with different imaging methods of 1.5 HI US $(n=42)$.

\begin{tabular}{lcc} 
Vascular phase & \multicolumn{2}{c}{ Post-vascular phase } \\
\cline { 2 - 3 } Enhancement & No & $\begin{array}{c}\text { Corona } \\
\text { enhancement }\end{array}$ \\
enhancement
\end{tabular}

\begin{tabular}{lccc}
\hline Positive cases & 36 & 42 & 30 \\
$(\%)$ & $(85.7)$ & $(100)$ & $(71.4)$ \\
\hline
\end{tabular}

(a)

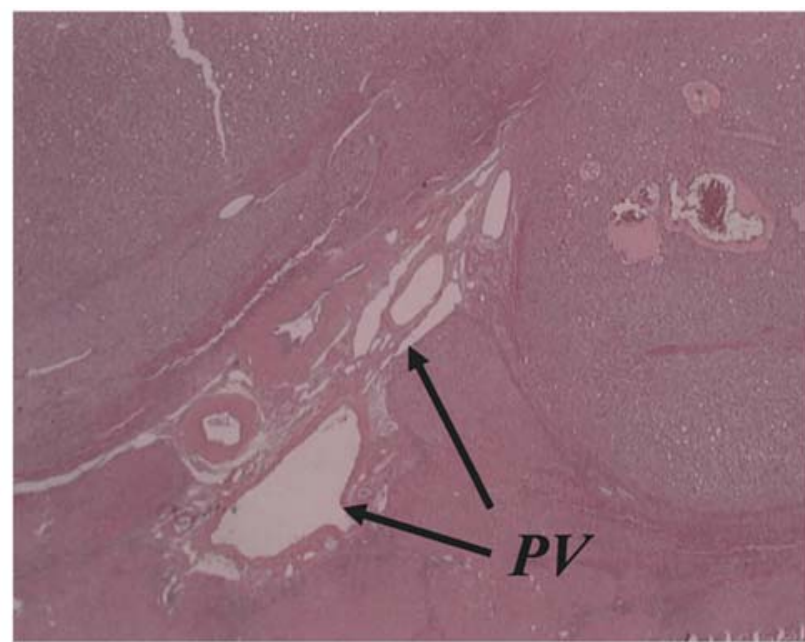

(b)

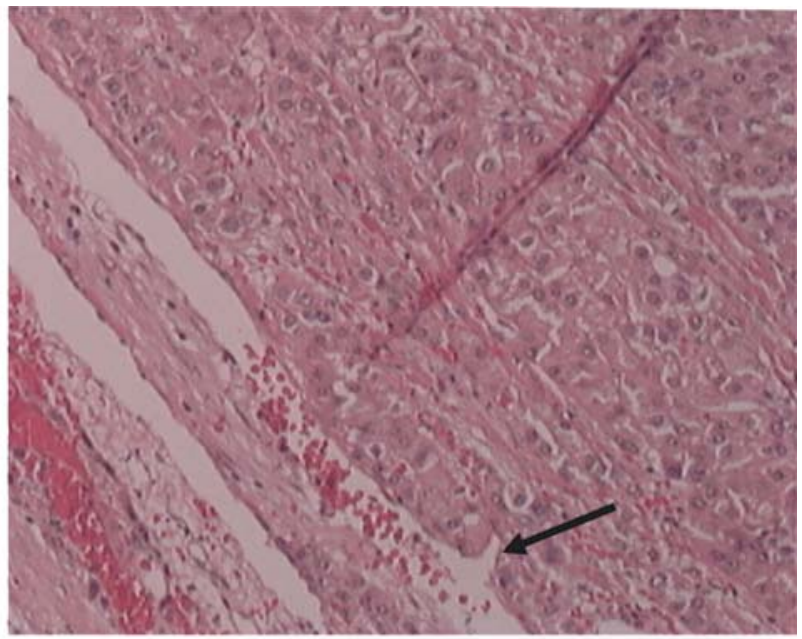

Imaging sonography.

\begin{tabular}{|c|c|c|}
\hline & $\begin{array}{l}\text { No. of } \\
\text { nodules }\end{array}$ & $\begin{array}{l}\text { 1.5 Harmonic Imaging sonography } \\
\text { (corona enhancement in } \\
\text { post-vascular phase) }(\%)\end{array}$ \\
\hline $\mathrm{HCC}$ & 42 & $30(71.4)$ \\
\hline Liver metastasis & 20 & $0 \quad(0)$ \\
\hline Hemangioma & 8 & $0 \quad(0)$ \\
\hline
\end{tabular}
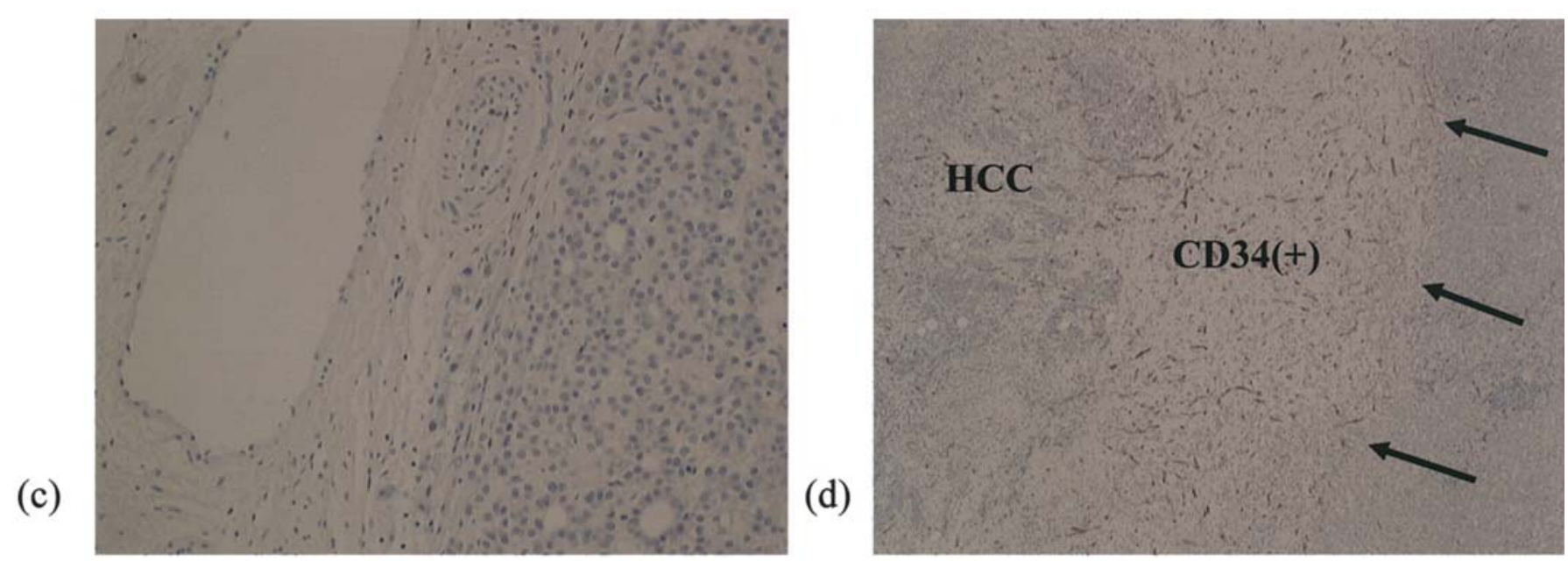

Figure 3. Histological examination of the margins of an HCC lesion (hematoxylin-eosin stain, original magnification x100). (a), histological demonstration of the dilated portal vessels surrounding the tumor (septal region) (arrow) (hematoxylin-eosin stain, original magnification x100). (b), histological section of the margins of the hepatic tumor, demonstrating continuity between the tumor sinusoids and the small vessels of the inner layer (arrow) (hematoxylin-eosin stain, original magnification x100). (c), in immunohistochemical staining (CD68), neither the tumor nor the surrounding hepatic parenchyma were stained, meaning no increase in Kupffer cells. (d), in immunohistochemical staining (CD34), CD34-positive endothelial cells were stained in surrounding areas of tumors (arrow).

examination of the margins of the hepatic tumors confirmed the continuity of the tumor sinusoids and the small vessels of the inner layer. Next, we examined the existence of Kupffer cells and endothelial cells using immunohistochemical staining because Levovist may be trapped by these cells. Immunohistochemical staining using anti-CD68 antibody, revealed few positive-staining cells in the tumor and surrounding tissue, demonstrating no increase in Kupffer cells. In contrast, CD34- positive cells are abundant in surrounding tumors, indicating a large number of endothelial cells in adjacent liver tissue (Fig. 3).

\section{Discussion}

In 1.5 HI US, images are obtained using a frequency band that is lower than that of the 2 nd harmonic components, 
thus significantly improving the non-uniformity of contrast enhancement that is frequently observed when a sector transducer is used. Furthermore, because images are acquired by selecting a band in which there are no tissue echoes, the contrast between the microbubbles and tissue is significantly improved. An additional extremely important advantage is related to the overall gain of the system.

In conventional examinations, if the gain is increased excessively, motion artifacts become noticeable in the tissue harmonic and pseudo-Doppler methods. In 1.5 HI US, on the other hand, since there are almost no tissue echoes, the gain can be increased without problems. As a result, contrast sensitivity is significantly improved in deep regions from which only weak echoes are received. In addition, images are generated using the B-mode processing system, resulting in good spatial resolution and minimal motion artifacts and blooming, which are often problems in the pseudo-Doppler method. Furthermore, the combined use of flash echo mode and monitor mode ensures that slices can be maintained or adjusted in real-time even though images are acquired at high acoustic power while the microbubbles are being destroyed. The enhanced slice can therefore be adjusted easily during the continuous injection of contrast agent. In 1.5 HI US, with the use of a frequency near the mean value, acceptable contrast enhancement is obtained from the near field to the far field. This method also avoids the problems specific to the Doppler method and provides clear contrast-enhanced images.

The results of the present clinical study clearly demonstrate that $1.5 \mathrm{HI}$ US is effective for evaluating the vascularity of HCCs, including the assessment of tumor vessels during continuous vascular phase arterial imaging as well as the assessment of post-vascular phase enhancement, staining of blood flow within tumors. In vascular phase real-time continuous imaging, the movement of microbubbles provides the signals for enhancement. In other words, the image obtained depicts the flow of the microbubbles into the tumor vessels. An additional finding in the post-vascular phase was that the tumor parenchyma was less strongly enhanced than the surrounding hepatic parenchyma, although some residual enhancement was seen. This is thought to reflect the flow of microbubbles out of the tumor during the specified interval, resulting in reduced enhancement.

In addition, the current study clearly demonstrated the strong corona enhancement of adjacent liver, in the areas surrounding the tumors in the post-vascular phase, after approximately $5 \mathrm{~min}$. This corona enhancement was not observed in metastatic liver tumors nor hepatic hemangiomas. Previous study revealed that the blood supply to HCC lesions is mainly via the hepatic artery. A single-level dynamic CTHA demonstrated that the blood entered the lesion via the hepatic artery, spread throughout the lesion, flowed into the portal venule in the adjacent liver, and then spread into the surrounding liver, forming corona enhancement at $\sim 30 \mathrm{sec}$ after contrast enhancement injection from hepatic artery. We hypothesized that it may be difficult to detect these blood drainage areas using an ultrasonographic technique. In this study, we observed similar corona enhancement using ultrasonography with contrast medium using 1.5 HI. However, this was observed at a more delayed phase, approximately 5 min after the injection, in ultrasonography than in CT.
In order to elucidate the mechanism of this corona enhancement, we performed histological analysis. Histological examination of 7 nodules confirmed the presence of dilated portal vessels surrounding the tumor (septal area) and also revealed that these portal branches were continuous from the veins within the tumor. In addition, immunohistochemical analysis revealed that CD34-positive endothelial cells but not CD68-positive Kupffer cells are abundant in areas surrounding the tumor. Although continuity with an outflow blood vessel of hepatocellular carcinoma is not proved, these contrast enhanced areas may be blood drainage areas from HCC. We speculated that the microbubble contrast may be trapped by a large number of endothelial cells, forming corona enhancement.

In conclusion, $1.5 \mathrm{HI}$ US permits the vascular structures of HCC and the blood flow dynamics within the tumor to be evaluated, and has been shown to be effective for the differential diagnosis of HCC. The corona enhancement observed in post-vascular phase images is thought to reflect the enhancement of endothelial cells in the blood drainage area. US corona enhancement is expected to be a useful finding for establishing the diagnosis of HCC and is also expected to improve the understanding of vascular dynamics within such tumors.

\section{References}

1. Choi BI, Kim TK, Han JK, et al: Vascularity of hepatocellular carcinoma: assessment with contrast-enhanced second-harmonic versus conventional power Doppler US. Radiology 214: 381-386, 2000.

2. Hosten N, Puls R, Lemke A-J, et al: Contrast enhanced power Doppler sonography: improved detection of characteristic flow patterns in focal liver lesions. J Clin Ultrasound 27: 107-115, 1999.

3. Ding H, Kudo M, Onda H, et al: Evaluation of posttreatment response of hepatocellular carcinoma with contrast-enhanced coded phase-inversion harmonic US: comparison with dynamic CT. Radiology 221: 721-730, 2001.

4. Heckemann RA, Cosgrove DO, Blomley MJK, et al: Liver lesions: intermittent second-harmonic gray-scale US can increase conspicuity with microbubble contrast material - early experience. Radiology 216: 592-596, 2000

5. Harvey CJ, Blomley MJK, Eckersley RJ, et al: Pulseinversion mode imaging of liver specific microbubbles: improved detection of subcentimetre metastases. Lancet 355: 807-808, 2000.

6. Blomley MJK, Albrecht T, Wilson SR, et al: Improved detection of metastatic liver lesions using pulse inversion harmonic imaging with Levovist: a multicenter study. Radiology 213: $491,1999$.

7. Burns PN, Wilson SR, Muradali D, et al: Microbubble destruction is the origin of Harmonic signals from FS 069. Radiology 201: 158-159, 1996.

8. Calliada F, Campani R, Bottinelli O, et al: Ultrasound contrast agents basic principals. Eur J Radiol 27: 157-160, 1998.

9. Wilson SR, Burns PN, Muradali D, et al: Harmonic hepatic US with microbubble contrast agents: initial experience showing improved characterization of hemangioma, hepatocellular carcinoma, and metastasis. Radiology 215: 153-161, 2000.

10. Blomley MJK, Albrecht T, Cosgrove DO, et al: Stimulated acoustic emission to image a late liver and spleen-specific phase of Levovist in normal volunteers and patients with and without liver disease.Ultrasound Med Biol 25: 1341-1352, 1999.

11. Blomley MJK, Sidhu PS, Cosgrove DO, et al: Do different types of liver lesions differ in their uptake of the microbubble contrast agent SH U 508A in the late liver phase? Early experience. Radiology 220: 661-667, 2001.

12. Kim TK, Choi BI, Han JK, et al: Hepatic tumors: contrast agentenhancement patterns with pulse-inversion harmonic US. Radiology 216: 411-417, 2000. 
13. Harvey CJ, Blomley MJK, Eckersley RJ, et al: Hepatic malignancies: improved detection with pulse-inversion US in late phase of enhancement with SH U 508A-early experience. Radiology 216: 903-908, 2000.

14. Yamamoto K, Shiraki K, Deguchi M, et al: Diagnosis of hepatocellular carcinoma using digital subtraction imaging with the contrast agent, Levovist: comparison with helical CT, digital subtraction angiography, and US angiography. Oncol Rep 9: 789-792, 2002.

15. Yamamoto K, Shiraki K, Nakanishi S, et al: The usefulness of digital subtraction imaging with Levovist in the diagnosis of focal hepatic tumors. Int J Oncol 22: 353-358, 2003.

16. Yamamoto K, Shiraki K, Nakanishi S, et al: Usefulness of digital subtraction imaging with Levovist in the diagnosis of hepatocellular carcinomas. Oncol Rep 13: 95-99, 2005.

17. Kim AY, Choi BI, Kim TK, et al: Hepatocellular carcinoma: power Doppler US with a contrast agent-preliminary results. Radiology 209: 135-140, 1998.

18. Kim TK, Han JK, Kim AY, et al: Limitations of characterization of hepatic hemangiomas using a sonographic contrast agent (Levovist) and power Doppler ultrasonography. J Ultrasound Med 18: 737-743, 1999.
19. Choi D, Lim HK, Kim SH, et al: Hepatocellular carcinoma treated with percutaneous radio-frequency ablation: usefulness of power Doppler US with a microbubble contrast agent in evaluating therapeutic response-preliminary results. Radiology 217: 558-563, 2000

20. Yamamoto K, Shiraki K, Nakanishi S, et al: The usefulness of 1.5 harmonic imaging ultrasonography with Levovist in the diagnosis of focal hepatic tumors. Int J Oncol 27: 989-995, 2005.

21. Yamamoto K, Shiraki K, Nakanishi S, et al: 1.5 Harmonic Imaging Sonography with microbubble contrast agent improves characterization of hepatocellular carcinoma. World J Gastroenterol 11: 5607-5613,2005.

22. Kawagishi T: Technical description of 1.5-Harmonic Imaging, an effective technique for contrast-enhanced ultrasound diagnosis. Medical Rev 87: 22-25, 2002.

23. Kawagishi T, Mine Y, Kamiyama N, et al: New imaging method for high MI contrast imaging: 1.5 Harmonic Imaging. J Ultrasound Med 29: 241, 2002 